\title{
CREATING RIGOROUS OPEN CLOAKS
}

\section{T. C. Han ${ }^{\dagger}$}

EHF Key Laboratory of Fundamental Science

School of Electronic Engineering

University of Electronic Science and Technology of China

Chengdu, Sichuan 611731, China

\section{C.-W. Qiu}

Department of Electrical and Computer Engineering National University of Singapore

Kent Ridge, Singapore 119620, Republic of Singapore

\section{H. Tang}

EHF Key Laboratory of Fundamental Science

School of Electronic Engineering

University of Electronic Science and Technology of China

Chengdu, Sichuan 611731, China

Abstract-Based on compressing coordinate transformation (CCT) that transforms a region into a smaller region, a rigorous method for designing a cloak with controllable open window is proposed. The open cloak consists of a portion of an ideal closed cloak and an independent system whose position and parameters can be controlled. The system could be circular or diamond-shaped with coordinate transformation in radius direction and one axis of Cartesian coordinates, respectively. This cloak is able to externally hide an arbitrary-shaped object with arbitrary material property including PEC, and the object is open to the outside space. Full-wave simulations validate this rigorous cloaking concept.

Received 27 June 2010, Accepted 29 July 2010, Scheduled 9 August 2010

Corresponding author: T. C. Han (tchan@uestc.edu.cn).

$\dagger$ Also with Department of Electrical and Computer Engineering, National University of Singapore, Kent Ridge, Singapore 119620, Republic of Singapore. 


\section{INTRODUCTION}

In the past few years, various efforts have been made on the realization of invisibility cloaks due to their exciting property of completely hiding objects from electromagnetic (EM) detection. Scientists have developed conformal mapping method [1] and coordinate transformation method [2], which can protect the cloaked object of arbitrary shape from EM radiation. General relativity is proposed as the theoretical tools for controlling EM fields [3]. The first practical realization of a cylindrical cloak at microwave frequencies has been presented by Schurig et al. [4]. Inspired by the above pioneering work, many further investigations on cloaking have been conducted, including the petal-shaped cloak [5], elliptical cloaks [6], slab cloaks [7], toroids cloak [8], cloak with a twin cavity [9], cloaks of twisted domains [10], arbitrary shaped two-dimensional cloak [11] and three-dimensional cloak [12]. Also a different simulation method based on FDTD [13] and the sensitivity of cloaks to several factors [14] have been carefully studied.

In the approaches mentioned above, the cloaks are closed, and the object is concealed in a cloaking shell. The open cloak [15] has been investigated by an approximation approach. This cloak itself has an open window based on the approximation that the cloak is infinitesimally thin in certain areas, and such discontinuity is thus ignored resulting in an effective open window. EM scattering still exists outside the open cloak since it is an approximate approach, which implies that the window cannot be large. Based on the concept of complementary media, the cloaks that can cloak objects at a distance outside the cloaking shell have been investigated $[16,17]$. Complementary media can be designed under a special kind of coordinate transformation, i.e., folding a piece of space into another. Using this concept, many further investigations have been conducted, including the pathway that can block EM waves but allow the passage of other entities [18], the hidden portal that can conceal an entrance from the EM wave detection [19], new strategy to conceal an object [20], guiding waves through an invisible tunnel [21], the cloak exhibiting magnified and shifted scattering wave [22], and equiscatterer [23]. Recently, another cloaking method has been proposed to design active exterior cloaks [24], which has the disadvantage that one needs to know in advance the incoming probing wave and its phase information.

In this paper, we propose a rigorous recipe for creating open cloaks, in contrast to the approximation adopted in [15]. The proposed concept is based on the combination of transformation optics and 
folded geometry to segment an original closed cloak [2] into two parts and replace one part by an independent system. Therefore, by assigning a few constitutions designed by the proposed method, EM invisibility can be achieved from this rigorous method without any approximation. Compared with closed cloak [2], the object cloaked by using this cloak is open to the outer space, making the matter or information exchange possible especially for moving objects. More importantly, this cloak does not rely on "anti-object" concept $[16,17]$ and is thus independent of the cloaked object, which greatly enhances the applicability of open cloaks.

\section{THEORETICAL ANALYSIS}

A schematic diagram illustrating the concept of the rigorous open cloak with a circular system is shown in Fig. 1. A closed cloak [1] placed at $\vec{r}_{0}$ in virtual space $\left(x^{\prime}, y^{\prime}, z^{\prime}\right)$ is divided into two portions in Fig. 1(a). The blue segment is kept invariant while the red segment is replaced with a circular system. Then an open cloak in the real space $(x, y, z)$ is created in Fig. 1(b). Fig. 1(c) shows a zoom-in view of the circular system in Fig. 1(b). It can be seen that the system is divided into three regions including region $\mathrm{I}(b<r<c)$ denoted by $\left(r_{1}, \theta_{1}, z_{1}\right)$, region II $(a<r<b)$ denoted by $\left(r_{2}, \theta_{2}, z_{2}\right)$, and region III $(0<r<a)$ denoted by $\left(r_{3}, \theta_{3}, z_{3}\right)$. We first compress region I, region II and region III into region III. Then the parameters of the green area (core material) and red area (image) can be obtained. The image in physical space corresponds to a portion of a closed cloak with $\varepsilon^{\prime}$ and $\mu^{\prime}[2]$ in virtual space. Secondly, the folded geometry is employed to compensate the
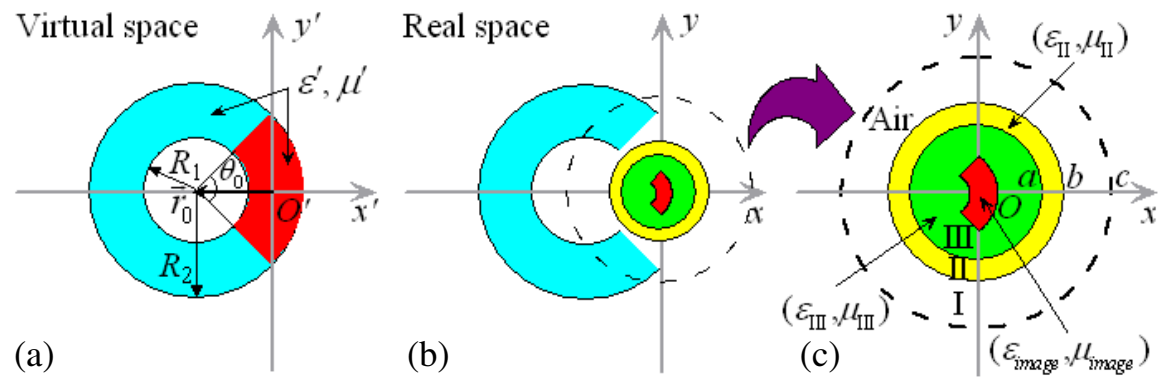

Figure 1. The scheme of designing the rigorous open cloak with a circular system. (a) A closed cloak [2] in virtual space is divided into two segments. (b) The red segment in (a) is replaced with a circular system (an open cloak is formed). (c) A magnified view of the system in (b). 
discontinuity by using CCT. Then the parameters of the yellow area (complementary material) can be obtained. Note that the permittivity and permeability are relative parameters throughout.

The transformation equations compressing region I, region II and region III into region III are

$$
r_{3}=\frac{a}{c} r^{\prime}, \quad \theta_{3}=\theta^{\prime}, \quad z_{3}=z^{\prime}
$$

The permittivity and permeability tensors become

$$
\varepsilon_{I I I}=\mu_{I I I}=\mathbf{A}^{c} \mathbf{A}^{c T} / \operatorname{det}\left(\mathbf{A}^{c}\right)
$$

where $\mathbf{A}_{i j}^{c}=\partial x_{3}^{i} / \partial x^{\prime j}$ is the Jacobian transformation matrix from Eq. (1).

Therefore, the permittivity and permeability of the core material are

$$
\varepsilon_{I I I}=\mu_{I I I}=\operatorname{diag}\left[1,1,(c / a)^{2}\right] .
$$

Assuming a closed cloak with the inner boundary $R_{1}$ and outer boundary $R_{2}$ [Fig. 1(a)], its parameters can be expressed as $\varepsilon_{r}^{\prime}\left(r^{\prime}\right)=$ $\mu_{r}^{\prime}\left(r^{\prime}\right)=\frac{\left|\overrightarrow{r^{\prime}}-\vec{r}_{0}\right|-R_{1}}{\left|\overrightarrow{r^{\prime}}-\vec{r}_{0}\right|}, \varepsilon_{\theta}^{\prime}\left(r^{\prime}\right)=\mu_{\theta}^{\prime}\left(r^{\prime}\right)=\frac{\left|\overrightarrow{r^{\prime}}-\vec{r}_{0}\right|}{\left|\overrightarrow{r^{\prime}}-\vec{r}_{0}\right|-R_{1}}, \varepsilon_{z}^{\prime}\left(r^{\prime}\right)=\mu_{z}^{\prime}\left(r^{\prime}\right)=$ $\frac{\left|\overrightarrow{r^{\prime}}-\vec{r}_{0}\right|-R_{1}}{\left|\overrightarrow{r^{\prime}}-\vec{r}_{0}\right|}\left(\frac{R_{2}}{R_{2}-R_{1}}\right)^{2}$. Then the parameters of its corresponding image in physical space become

$$
\varepsilon_{\text {image }}=\mu_{\text {image }}=\mathbf{A}^{c} \cdot \varepsilon^{\prime}\left(r_{3}\right) \cdot \mathbf{A}^{c T} / \operatorname{det}\left(\mathbf{A}^{c}\right)
$$

where $\varepsilon_{r}^{\prime}\left(r_{3}\right)=\frac{\left|\vec{r}_{3}-a \vec{r}_{0} / c\right|-a R_{1} / c}{\left|\vec{r}_{3}-a \vec{r}_{0} / c\right|}, \varepsilon_{\theta}^{\prime}\left(r_{3}\right)=\frac{1}{\varepsilon_{r}^{\prime}\left(r_{3}\right)}$, and $\varepsilon_{z}^{\prime}\left(r_{3}\right)=\varepsilon_{r}^{\prime}\left(r_{3}\right)$. $\left(\frac{R_{2}}{R_{2}-R_{1}}\right)^{2}$.

In order to compensate the discontinuity by using CCT, the folded geometry is adopted. The transformation equations folding region I into region II are

$$
r_{2}=\frac{a-b}{c-b}\left(r_{1}-b\right)+b, \quad \theta_{2}=\theta_{1}, \quad z_{2}=z_{1} .
$$

The permittivity and permeability tensors become

$$
\varepsilon_{I I}=\mu_{I I}=\mathbf{A}^{f} \mathbf{A}^{f T} / \operatorname{det}\left(\mathbf{A}^{f}\right)
$$

where $\mathbf{A}_{i j}^{f}=\partial x_{2}^{i} / \partial x_{1}^{j}$ is the Jacobian transformation matrix from Eq. (5).

Thus, the parameters of the complementary material in polar coordinates are

$$
\varepsilon_{I I}=\mu_{I I}=\operatorname{diag}\left[\frac{k_{1} r_{2}-k_{2} b}{k_{1} r_{2}}, \frac{k_{1} r_{2}}{k_{1} r_{2}-k_{2} b}, \frac{k_{1}\left(k_{1} r_{2}-k_{2} b\right)}{r_{2}}\right]
$$


where $k_{1}=\frac{c-b}{a-b}$ and $k_{2}=\frac{c-a}{a-b}$.

The circular system discussed above is constructed by operating CCT along the radial direction. The system can also be designed by operating CCT along one axis of Cartesian coordinates [25], which is thus in the diamond shape. A schematic diagram illustrating the concept of a rigorous open cloak with diamond-shaped system is shown in Fig. 2. In Fig. 2(c), we can find that the system is divided into three regions including region I denoted by $\left(x_{1}, y_{1}, z_{1}\right)$, region II denoted by $\left(x_{2}, y_{2}, z_{2}\right)$, and region III denoted by $\left(x_{3}, y_{3}, z_{3}\right)$.

The transformation equations compressing region I, region II and region III into region III are

$$
x_{3}=x^{\prime}, \quad y_{3}=\frac{a}{c} y^{\prime}, \quad z_{3}=z^{\prime} .
$$

Therefore, the permittivity and permeability of the core material are

$$
\varepsilon_{I I I}=\mu_{I I I}=\operatorname{diag}[c / a, a / c, c / a]
$$

Assuming a closed cloak with the inner boundary $R_{1}$ and outer boundary $R_{2}$ [Fig. 2(a)], its parameters can be expressed as

$$
\varepsilon^{\prime}\left(x^{\prime}, y^{\prime}\right)=\mu^{\prime}\left(x^{\prime}, y^{\prime}\right)=\left(\begin{array}{ccc}
\varepsilon_{x x}^{\prime} & \varepsilon_{x y}^{\prime} & 0 \\
\varepsilon_{y x}^{\prime} & \varepsilon_{y y}^{\prime} & 0 \\
0 & 0 & \varepsilon_{z z}^{\prime}
\end{array}\right)
$$

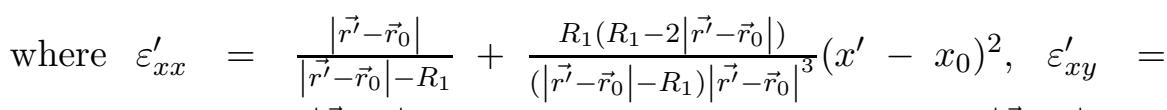
$\varepsilon_{y x}^{\prime}=\frac{R_{1}\left(R_{1}-2\left|\overrightarrow{r^{\prime}}-\vec{r}_{0}\right|\right)}{\left(\left|\overrightarrow{r^{\prime}}-\vec{r}_{0}\right|-R_{1}\right)\left|\overrightarrow{r^{\prime}}-\vec{r}_{0}\right|^{3}}\left(x^{\prime}-x_{0}\right)\left(y^{\prime}-y_{0}\right), \quad \varepsilon_{y y}^{\prime}=\frac{\left|\overrightarrow{r^{\prime}}-\vec{r}_{0}\right|}{\mid \overrightarrow{r^{\prime}-\vec{r}_{0} \mid-R_{1}}}+$
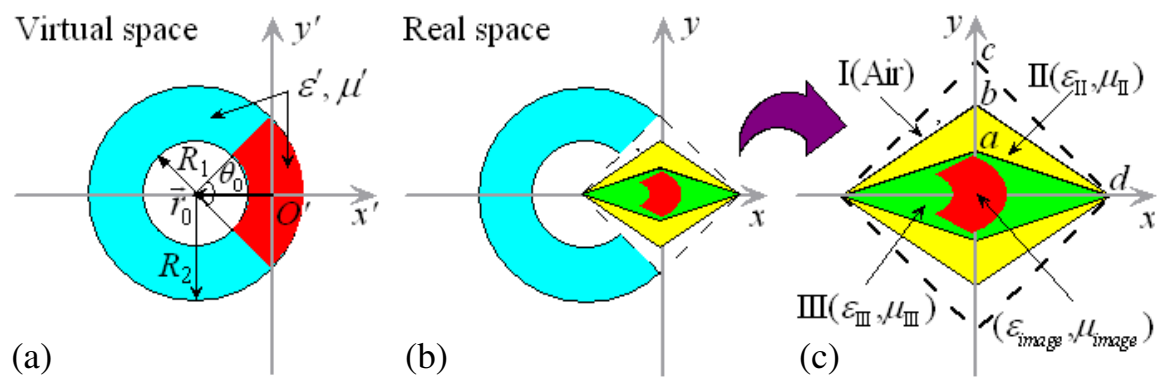

Figure 2. The scheme of designing an open cloak with a diamondshaped system. (a) A closed cloak [2] in virtual space is divided into two segments. (b) The red segment in (a) is replaced with a diamondshaped system to construct the open cloak. (c) The magnified view of the system in (b). 
$\frac{R_{1}\left(R_{1}-2\left|\overrightarrow{r^{\prime}}-\vec{r}_{0}\right|\right)}{\left(\left|\overrightarrow{r^{\prime}}-\vec{r}_{0}\right|-R_{1}\right)\left|\overrightarrow{r^{\prime}}-\vec{r}_{0}\right|^{3}}\left(y^{\prime}-y_{0}\right)^{2}, \quad \varepsilon_{z z}^{\prime}=\left(\frac{R_{2}}{R_{2}-R_{1}}\right)^{2} \frac{\left|\overrightarrow{r^{\prime}}-\vec{r}_{0}\right|-R_{1}}{\left|\overrightarrow{r^{\prime}}-\vec{r}_{0}\right|},\left|\overrightarrow{r^{\prime}}\right|=$ $\sqrt{x^{\prime 2}+y^{\prime 2}}$, and $\left|\overrightarrow{r^{\prime}}-\vec{r}_{0}\right|=\sqrt{\left(x^{\prime}-x_{0}\right)^{2}+\left(y^{\prime}-y_{0}\right)^{2}}$.

Then the parameters of its corresponding image in physical space become

$$
\varepsilon_{\text {image }}=\mu_{\text {image }}=\mathbf{A} \cdot \varepsilon^{\prime}\left(x_{3}, y_{3}\right) \cdot \mathbf{A}^{T} / \operatorname{det}(\mathbf{A})
$$

where $\mathbf{A}=\operatorname{diag}[1, a / c, 1]$, and $\varepsilon^{\prime}\left(x_{3}, y_{3}\right)$ can be obtained by substituting (8) into (10).

The transformation equations folding region I into region II are

$$
x_{2}=x_{1}, \quad y_{2}=w_{1}\left(d-x_{1}\right)-w_{2} y_{1}, \quad z_{2}=z_{1} .
$$

where $w_{1}=\frac{b(c-a)}{d(c-b)}$ and $w_{2}=\frac{b-a}{c-b}$.

Thus, the parameters of the complementary material are

$$
\varepsilon_{I I}=\mu_{I I}=\left(\begin{array}{ccc}
-1 / w_{2} & w_{1} / w_{2} & 0 \\
w_{1} / w_{2} & -w_{1}^{2} / w_{2}-w_{2} & 0 \\
0 & 0 & -1 / w_{2}
\end{array}\right)
$$

\section{SIMULATIONS AND DISCUSSION}

To verify the concept of designing rigorous open cloaks, we make full-wave simulations based on the finite element method (FEM). The working frequency is $0.5 \mathrm{GHz}$ under TE polarization, and we set $R_{1}=0.4 \mathrm{~m}$ and $R_{2}=0.8 \mathrm{~m}$.

In Fig. 3, we consider a PEC with the radius $0.3 \mathrm{~m}$ covered by an open cloak with a circular system, and the axis of the PEC is located at $x=-0.6 \mathrm{~m}$ and $y=0 \mathrm{~m}$. Clearly, it can be observed that the open cloak is able to guide the arbitrarily incident plane waves to propagate around the center region and the EM wave fronts remain planar after the cloak. As expected, the absence of scattered waves validates the cloaking concept proposed in this paper. Note that the spatial transformation in Fig. 3 is operated along the radial direction.

We also calculated the electric fields of the open cloak with a diamond-shaped system, in which the spatial transformation is operated along $y$-axis. In Fig. 4, we consider an angular PEC with the radius $0.4 \mathrm{~m}$ covered by an open cloak with a diamond-shaped system, and the axis of the PEC is located at $x=-0.6 \mathrm{~m}$ and $y=0 \mathrm{~m}$. Fig. 4(a) corresponds to the plane wave incident from the left to the right, and Fig. 4(b) corresponds to the plane wave incident from the bottom to the top. In both cases, we can observe that the scattered waves are reduced significantly and the wave fronts are maintained in the presence of the open cloak. It is clear that perfect open cloaks can be achieved. 

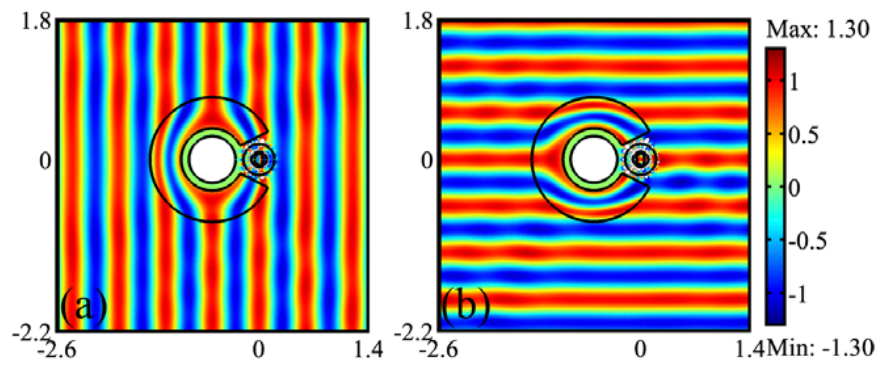

Figure 3. The snapshots of total electric fields for an open cloak with a circular system. The system follows the design of $a=0.1 \mathrm{~m}$, $b=0.2 \mathrm{~m}, c=0.4 \mathrm{~m}$, and $\theta_{0}=90^{\circ}$. (a) The incident plane wave is propagating in the horizontal direction. (b) The incident plane wave is propagating in the vertical direction.
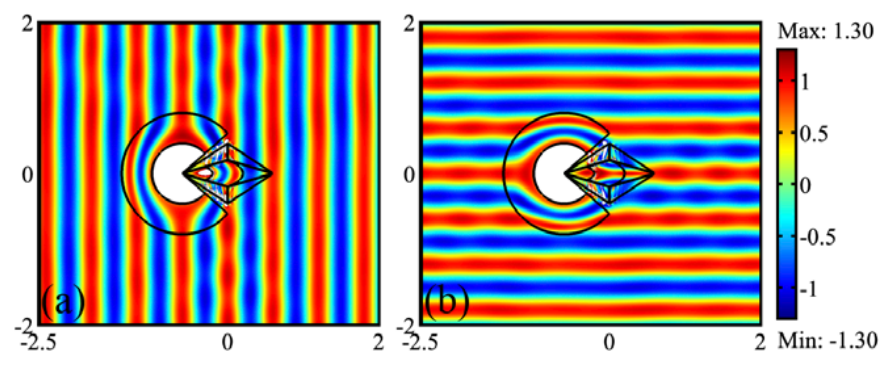

Figure 4. The snapshots of total electric fields for an open cloak with a diamond-shaped system. The system follows the design of $d=0.6 \mathrm{~m}$, $c=\sqrt{0.8^{2}-0.6^{2}} \mathrm{~m}, b=3 c / 4, a=c / 3, \theta_{0}=82.8^{\circ}$. (a) The plane wave is incident in the horizontal direction. (b) The plane wave is incident in the vertical direction.

\section{CONCLUSION}

In this paper, a rigorous method of designing open cloak is proposed. The open cloak can be designed with a circular or diamondshaped system. It performs as perfectly as the original closed cloak. In contrast to the closed cloak [2], the object cloaked by such an open cloak contains multiple open windows which are controllable. Compared to the approximate open cloak [15], the proposed open cloak is designed analytically and rigorously without any assumptions. Compared to the "anti-object" cloak [16], the proposed open cloak can be repeatedly used for various objects without removing, rebuilding, or refilling different anti-objects in different 
shapes into the complementary layer each time. Simulation results validate the design method, and this method can be easily extended to design open cloaks for arbitrarily-shaped closed cloaks.

\section{REFERENCES}

1. Leonhardt, U., "Optical conformal mapping," Science, Vol. 312, No. 5781, 1777-1780, 2006.

2. Pendry, J. B., D. Schurig, and D. R. Smith, "Controlling electromagnetic fields," Science, Vol. 312, No. 5781, 1780-1782, 2006.

3. Leonhardt, U. and T. G. Philbin, "General relativity in electrical engineering," New J. Phys., Vol. 8, No. 10, 247, 2006.

4. Schurig, D., J. J. Mock, B. J. Justice, S. A. Cummer, J. B. Pendry, A. F. Starr, and D. R. Smith, "Metamaterial electromagnetic cloak at microwave frequencies," Science, Vol. 314, No. 5801, $977-$ 980, 2006.

5. Han, T. C., X. H. Tang, and F. Xiao, "The petal-shaped cloak," Journal of Electromagnetic Waves and Applications, Vol. 23, No. 14-15, 2055-2062, 2009.

6. Jiang, W. X., T. J. Cui, X. M. Yang, Q. Cheng, R. P. Liu, and D. R. Smith, "Invisibility cloak without singularity," Appl. Phys. Lett., Vol. 93, 194102, 2008.

7. Chen, H. and C. T. Chan, "Electromagnetic wave manipulation by layered systems using the transformation media concept," Phys. Rev. B, Vol. 78, No. 5, 054204, 2008.

8. You, Y., G. W. Kattawar, and P. Yang, "Invisibility cloaks for toroids," Opt. Express, Vol. 17, No. 8, 6591-6599, 2009.

9. Chen, T. Y. and C. N. Weng, "Invisibility cloak with a twin cavity," Opt. Express, Vol. 17, No. 10, 8614-8620, 2009.

10. Schmiele, M., C. Rockstuhl, and F. Lederer, "Strategy for cloaking of twisted domains," Phys. Rev. A, Vol. 79, No. 5, 053854, 2009.

11. Hu, J., X. Zhou, and G. Hu, "Design method for electromagnetic cloak with arbitrary shapes based on Laplace's equation," Opt. Express, Vol. 17, No. 3, 1308-1320, 2009.

12. Cheng, Q., W. X. Jiang, and T. J. Cui, "Investigations of the electromagnetic properties of three-dimensional arbitrarily-shaped cloaks," Progress In Electromagnetics Research, Vol. 94, 105-117, 2009.

13. Silva-Macedo, J. A., M. A. Romero, and B. H. V. Borges, "An extended FDTD method for the analysis of electromagnetic field 
rotations and cloaking devices," Progress In Electromagnetics Research, Vol. 87, 183-196, 2008.

14. Zhang, J. J., Y. Luo, H. Chen, and B.-I. Wu, "Sensitivity of transformation cloak in engineering," Progress In Electromagnetics Research, Vol. 84, 93-104, 2008.

15. Ma, H., S. B. Qu, Z. Xu, and J. F. Wan, "The open cloak," Appl. Phys. Lett., Vol. 94, 103501, 2009.

16. Lai, Y., H. Y. Chen, Z. Q. Zhang, and C. T. Chan, "Complementary media invisibility cloak that cloaks objects at a distance outside the cloaking shell," Phys. Rev. Lett., Vol. 102, No. 9, 093901, 2009.

17. Chen, H. Y. and C. T. Chan, "Cloaking at a distance' from folded geometries in bipolar coordinates," Opt. Lett., Vol. 34, No. 17, 2649-2651, 2009.

18. Chen, H. Y., C. T. Chan, S. Y. Liu, and Z. F. Lin, "A simple rout to a tunable electromagnetic gateway," New J. Phys., Vol. 11, No. 8, 083012, 2009.

19. Luo, X. D., T. Yang, Y. W. Gu, H. Y. Chen, and H. R. Ma, "Conceal an entrance by means of superscatterer," Appl. Phys. Lett., Vol. 94, 223513, 2009.

20. Luo, Y., J. Zhang, H. Chen, B.-I. Wu, and J. A. Kong, "A new strategy to conceal an object from electromagnetic wave," Physical Optics, 2008.

21. Zhang, J., Y. Luo, H. Chen, J. Huangfu, B.-I. Wu, L. Ran, and J. A. Kong, "Guiding waves through an invisible tunnel," Opt. Express, Vol. 17, No. 8, 6203-6208, 2009.

22. Luo, Y., J. Zhang, H. Chen, B.-I. Wu, and L.-X. Ruan, "Wave and ray analysis of a type of cloak exhibiting magnified and shifted scattering effect," Progress In Electromagnetics Research, Vol. 95, 167-178, 2009.

23. Cojocaru, E., "Equiscatterer with complementary coordinatetransformed cylindrical media," J. Opt. Soc. Am. B, Vol. 27, No. 5, 895-898, 2010.

24. Vasquez, F. G., G. W. Milton, and D. Onofrei, "Active exterior cloaking for the 2D Laplace and Helmholtz equations," Phys. Rev. Lett., Vol. 103, No. 7, 073901, 2009.

25. Han, T. C., X. H. Tang, and F. Xiao, "External cloak with homogeneous material," J. Phys. D: Appl. Phys., Vol. 42, No. 23, 235403, 2009. 